\title{
Effets des sécheresses sur l'hydrologie de surface
}

\author{
Jacques Sircoulon \\ Institut français de recherche scientifique pour le développement en coopération
}

\section{Introduction}

L'opinion est régulièrement sensibilisée à l'impact des aléas climatiques sur la vie de la planète ; les moyens actuels des médias permettent à chaque foyer de suivre pratiquement en direct les effets extrêmes de la variabilité spatiotemporelle du climat : vagues de chaleur ou de froid, inondations, sécheresses. Les effets de la raréfaction de l'eau peuvent être aussi spectaculaires que ses excès : cours d'eau asséchés, barrages vides, cultures brûlées par le soleil, troupeaux décimés... L'aspect volontiers catastrophiste de l'information et le manque de recul pour apprécier la rareté du phénomène décrit entraînent un malaise diffus mais souvent volatil du lecteur. Sous la pression des circonstances, les organismes officiels et les pouvoirs publics prennent des mesures et débloquent des crédits, mais avec le retour des pluies le soulagement puis l'oubli s'installent trop souvent. Ceci correspond au fameux cycle de O.H. Foehner (Sécheresse $\rightarrow$ Prise de Conscience $\rightarrow$ Inquiétude $\rightarrow$ Panique $\rightarrow$ Pluie $\rightarrow$ Apathie $\rightarrow$ nouvelle sécheresse). Il est clair que s'il est impossible à l'heure actuelle de prévoir les sécheresses, sauf à court terme, les effets des sécheresses sont eux tout à fait évaluables et peu de plans de lutte contre la sécheresse à long terme survivent à l'érosion du temps.

Une des raisons de cette situation est peut-être due à l'aspect protéiforme de la sécheresse, diversement ressentie suivant le type de ressources affectées et le type d'activité économique le plus touché. Ainsi par exemple, un déficit de précipitation survenant à une période critique de végétation sera une véritable sécheresse pour les agriculteurs et non pour les producteurs d'électricité si l'hiver précédent a été assez pluvieux pour remplir les barrages en montagne.
Ce n'est que dans les cas les plus extrêmes où, par la durée et l'extension du déficit, tous les usages de l'eau sont affectés que l'on a affaire à la " grande sécheresse " ressentie par tous comme une catastrophe. Ce phénomène est assez rare en zone tempérée (1921, 1949, 1976 en Europe occidentale) mais est malheureusement beaucoup plus fréquent en zone aride (cas du Sahel en particulier).

\section{Quelques remarques sur les sécheresses hydrolo- giques}

On peut considérer qu'il y a sécheresse lorsqu'il se produit pendant un temps assez long (mois, année, suite d'années) et sur une superficie notable (plusieurs dizaines de milliers de $\mathrm{km}^{2}$ au moins) un déficit des disponibilités naturelles en eau (précipitations, écoulements de surface, eaux souterraines).

On ne retiendra dans ce document que les eaux de surface, en utilisant le déficit de précipitation comme un facteur de la sécheresse hydrologique. Les effets du déficit pluviométrique seront très divers suivant la période où se produit celui-ci, sa durée et sa sévérité. Il est clair que la notion de régime climatique est fondamentale : en terme de sécheresse climatique certains pays considèrent qu'il y a sécheresse lorsque le total pluviométrique sur 15 jours consécutifs est inférieur à un certain seuil, disons de $5 \mathrm{~mm}$; il est certain qu'en zone tropicale une telle définition n'a aucun sens.

Il est donc nécessaire d'associer déficit pluviométrique et type de régime climatique (donc hydrologique) pour évaluer les effets de cette diminution.

\section{Effects of droughts on surface hydrology}

After some thoughts on the characteristics of droughts from the hydrological point of view, the great historical droughts will be remembered that affected some temperate and tropical regions of our planet. The effects of droughts on surface water resources, both in temperate and tropical zones are analysed using recent events (1976 in Europe, 1968-1988 in the Sahel). 


\subsection{Cas de la France}

On peut schématiquement limiter à trois les types de climat :

Climat tempéré océanique : Il est caractérisé par des pluies réparties sur toute l'année mais avec une évapotranspiration élevée pendant six mois de l'année.

Il lui correspond un régime hydrologique pluvial avec maximum d'écoulement en hiver (au sens large) et minimum en été.

- Cas du déficit en été : crues inchangées, étiages plus accentués, module annuel un peu déficitaire.

- Cas du déficit en hiver : crues plus faibles, étiages plus longs et plus prononcés, module annuel très déficitaire.

Climat montagnard: Il est caractérisé par des températures plus basses en hiver et une rétention nivale de plusieurs mois.

Il lui correspond un régime hydrologique pluvio-nival avec un (ou deux) maximum d'écoulement, le plus prononcé étant au printemps et un minimum en été. Beaucoup plus rarement on trouve sur de petits bassins un régime glaciaire caractérisé par un minimum d'hiver et un maximum d'été.

- Cas du déficit en été : celui-ci est souvent associé à des températures plus élevées que la normale (ensoleillement prolongé) qui augmentent la fusion nivoglaciaire, d'où un module annuel peu affecté (VIVIAN, 1979).

- Cas du déficit en hiver : le manteau neigeux plus faible entraînera un module annuel déficitaire et une crue de printemps plus réduite.

Climat méditerranéen: Il est caractérisé par des précipitations abondantes en automne et secondairement au printemps et par une saison sèche d'été. Le régime hydrologique méditerranéen est défini par des crues parfois violentes en automne et au printemps et des étiages très marqués en été, avec souvent un assec prolongé.

— Cas du déficit en été : crues inchangées, étiages plus accentués ou assec plus durable, module annuel quasiment inchangé.

- Cas du déficit en hiver (sens large) : crues plus faibles (mais pas nécessairement sur les petits bassins), étiages plus précoces et très prononcés, module annuel plus faible (mais fonction de la répartition des pluies).

\subsection{Cas de la zone tropicale (exemple du Sahel)}

Il existe deux saisons très tranchées : une saison sèche de sept à neuf mois sans pratiquement pas de précipitations, une saison humide (saison des pluies) correspondant à notre été. Le régime hydrologique est marqué par une saison de hautes eaux de quelques mois, avec des débits faibles ou nuls le restant de l'année.

La sécheresse hydrologique dépendra de l'abondance de la saison des pluies et de la répartition des précipitations au cours de celle-ci ; un même total pluviométrique annuel pouvant ainsi donner un écoulement annuel allant du simple au double ou davantage suivant que les pluies sont trop espacées ou au contraire très groupées.

\subsection{Termes hydrologiques caractérisant ce type de séche- resse}

Les remarques qui précèdent montrent qu'il n'y a pas forcément concordance entre sécheresse pluviométrique et sécheresse hydrologique et que même si cette dernière est effective, les termes pouvant la caractériser ne semblent pas équivalents ni toujours représentatifs de la réalité.

La sécheresse hydrologique est le plus souvent quantifiée par les termes suivants :

- au niveau de l'année, de la saison, voire du mois: l'expression la plus courante est celle de l'hydraulicité qui représente, pour la durée de base considérée, le rapport du débit moyen $Q_{M}$ de l'année étudiée au débit moyen interannuel $Q_{I}$; on peut s'intéresser au déficit absolu exprimé en $\mathrm{m}^{3} / \mathrm{s}$, soit $Q_{M}-Q_{I}$, ou au déficit relatif exprimé en $\%$, soit $\left(Q_{M}-Q_{I}\right) / Q_{I}$. D'autres préféreront l'utilisation de la fréquence correspondante ou bien, étant connus la moyenne et l'écart-type de l'échantillon total, l'écart à la moyenne exprimé en fraction d'écart-type; les variantes sont nombreuses et constituent autant d'indices de sécheresse ;

- au niveau du débit journalier (ou de $n$ débits consécutifs ou non)

- débit minimum instantané journalier absolu $\left(\mathrm{m}^{3} / \mathrm{s}\right):$ on peut étudier la distribution statistique de l'ensemble des débits de l'échantillon et voir comment se place le débit de l'année étudiée. En fait cette notion est souvent sujette à caution: elle ne peut s'appliquer qu'au cours d'eau à l'écoulement pérenne ou s'asséchant rarement (l'étude du nombre de jours sans écoulement est alors intéressante) et est souvent peu significative si le tarage est difficile ou l'écoulement influencé. Aussi lui préfère-t-on en général les paramètres suivants :

- débit moyen le plus faible sur 10 (ou $n$ ) jours consécutifs ;

- débit non dépassé pendant 10 (ou $n$ ) jours les plus faibles consécutivement.

Cette liste n'est pas exhaustive, on peut aussi s'intéresser par exemple à l'effet de la sécheresse sur les crues, leur forme, les débits ou les niveaux atteints ou dépassés pendant $n$ jours consécutifs... ; la littérature abondante à ce sujet est le reflet des multiples utilisations de l'eau.

Nous citerons pour terminer ce point les six aspects de sécheresse caractérisés par BERAN et RODIER dans leur rapport OMM-UNESCO et qui reprennent certaines des notions évoquées plus haut :

- un déficit de l'écoulement superficiel d'une durée de trois semaines à trois mois pendant la période de germination et de croissance des plantes (intéresse notamment 
les cultures sèches ou les cultures irriguées sans réservoir de régularisation);

- des débits inférieurs de façon significative aux valeurs moyennes pendant la période de basses eaux (alimentation des villages et de certaines villes);

- un déficit significatif du débit moyen annuel (concerne la production d'énergie hydroélectrique ou l'irrigation avec de grands réservoirs);

- un niveau maximum annuel du cours d'eau nettement en dessous de la valeur moyenne de ces niveaux maximaux (concerne des irrigations sans pompage);

- une sécheresse continue pendant plusieurs années (seccas du Nordeste du Brésil et de certaines zones de l'Australie);

- une baisse significative du niveau des aquifères dans l'hypothèse où le volume annuel d'eau qu'on en extrait reste constant et voisin du volume correspondant à l'équilibre de la nappe (hypothèse toute théorique car lorsqu'il y a sécheresse il y a surexploitation des nappes).

\section{La sécheresse en zone tempérée}

Nous nous bornerons pour l'essentiel à l'Europe occidentale en présentant seulement quelques exemples qui donneront bien évidemment une vue limitée du phénomène.

\subsection{Historique}

Les données historiques antérieures aux réseaux de mesure sont en général moins riches que pour les crues des grands cours d'eau. Les archives anciennes peuvent parfois fournir des renseignements qualitatifs directs : assèchement d'un cours d'eau (comme la Sarthe en 1168 !), le tarissement des sources (1676 à 1686 dans le Midi de la France), la traversée à pied ou à cheval d'un fleuve (cas des années 1540 ou 1797 à 1807 selon les chroniques des villes riveraines du Rhin), arrêt de la batellerie sur un fleuve (la Tamise en 1666).

Le plus souvent les renseignements sont d'ordre indirect par le biais des calamités agricoles, des grandes chaleurs, des vendanges précoces ou des actions de grâce pour faire revenir les pluies (à sainte Sabine!).

LE Roy LADURIE (1983) nous cite ainsi la vague de chaleur en France de 1635 à 1639, l'année 1666 si sèche qu'elle favorisa l'incendie de Londres, la nuée de sauterelles dans le Midi de la France en $1686 \ldots$ Bien sûr, cette information est ponctuelle, souvent très subjective et il est difficile de trouver des relations sûres entre les dégâts agricoles ou sociaux et les précipitations ou l'écoulement. Un certain nombre d'auteurs se sont attachés à reconstituer des séquences de température ou de pluie sur plusieurs siècles en s'appuyant en particulier sur la dendrochronologie (aux Etats-Unis notamment).

Du point de vue quantitatif, si l'on excepte quelques stations anciennes suivies sans interruption (comme pour la pluviométrie l'observatoire de Genève créé en 1753, celui d'Exeter ouvert en 1820 ou celui de Marseille en 1821 ou comme la station hydrométrique du Rhin à Bâle existant depuis 1809) il faut attendre le début de la seconde moitié du $19^{\mathrm{e}}$ siècle pour voir en France apparaître de véritables réseaux de mesure (service hydrométrique de la Seine en 1854, réseau d'observations météorologiques créé par le Verrier en $1855 \ldots$...

BERAN (1985) a constitué un index de sécheresse hydrologique depuis 1850, en s'appuyant sur un échantillon de cours d'eau européens et en se basant sur la proportion de ceux-ci présentant en été des conditions quinquennales sèches. Il a dégagé ainsi pour l'Europe de l'Ouest des années où la sécheresse a présenté des conditions remarquables d'extension : 1857, 1863, 1872, 1911, 1921, 1934, $1947,1949,1964,1973$. Il faut bien sûr rajouter à cette liste l'année 1976 et pour la France l'automne 78, les années 85 et 86 dans le Sud-Ouest et l'hiver 88-89. Enfin pour souligner «l'actualité » des sćcheresses, il est peut-être bon de rappeler que l'année 1988 a été aux Etats-Unis la pire connue depuis cinquante ans, dépassant même 1976 en sévérité.

\subsection{Quelques exemples d'années très sèches}

On peut ainsi recenser en France depuis le début du $20^{\mathrm{C}}$ siècle une bonne dizaine d'années ayant connu des conditions climatiques propices au développement de sécheresses hydrologiques marquées affectant de vastes régions voire le pays tout entier.

Il n'existe pas actuellement de synthèse générale nationale fournissant les éléments statistiques ou les bilans de ces sécheresses en l'absence d'une mise à jour des grandes monographies régionales. Nous avons choisi de donner ici des commentaires, qui restent très fragmentaires, sur les années 1921, 1949 et 1976 qui ont connu une sécheresse hydrologique exceptionnellement sévère et étendue à une grande partie de l'Europe.

\subsubsection{La sécheresse de 1921}

Le déficit pluviométrique est remarquable pour trois raisons :

- il est très accentué puisque des minimums absolus sont relevés à de nombreuses stations ;

- il couvre une période très longue qui va de la fin de l'automne 1920 à la fin de l'hiver 1922;

- il couvre de nombreux pays, concernant aussi bien le sud (année record en Italie, seulement dépassée par la suite en 1945) que le nord (Belgique, Grande-Bretagne voir fig. 1) (page suiv.) ou l'Europe continentale (Tchécoslovaquie).

Tous les grands cours d'eau européens sont affectés, ainsi :

- la Tamise à Teddington (suivie depuis 1883) connaît les débits mensuels les plus faibles sur 2 à 9 mois consécutifs ; - le Rhin à Lobith (à son arrivée aux Pays-Bas, suivi depuis 1901) connaît le plus faible module annuel à ce jour avec un débit de $1096 \mathrm{~m}^{3} / \mathrm{s}$ soit un déficit de $52 \%$;

- le Danube à Vienne (dont nous connaissons les débits depuis 1901) présente un module de $1419 \mathrm{~m}^{3} / \mathrm{s}$ (module interannuel de $1990 \mathrm{~m}^{3} / \mathrm{s}$ sur 80 ans). Il faudra attendre l'année 1972 pour observer un débit annuel plus faible ; 


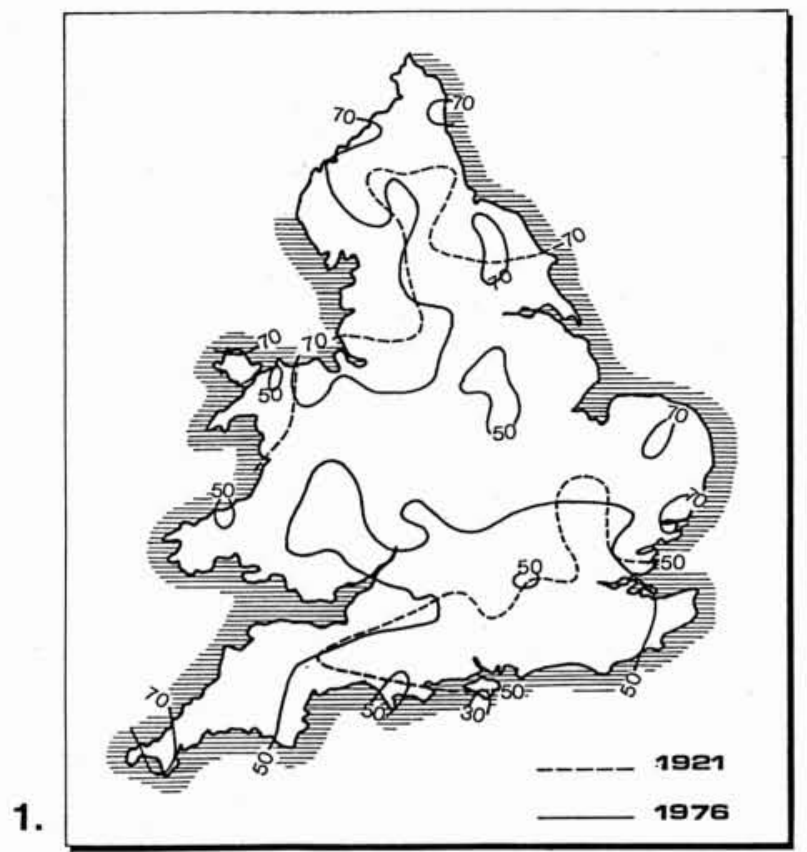

Pourcentage des précıpitations - par rapport à la moyenne interannuelle en Angleterre et au Pays de Galles.

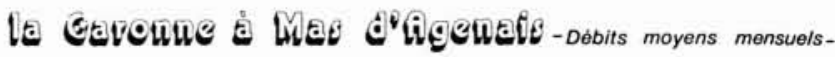

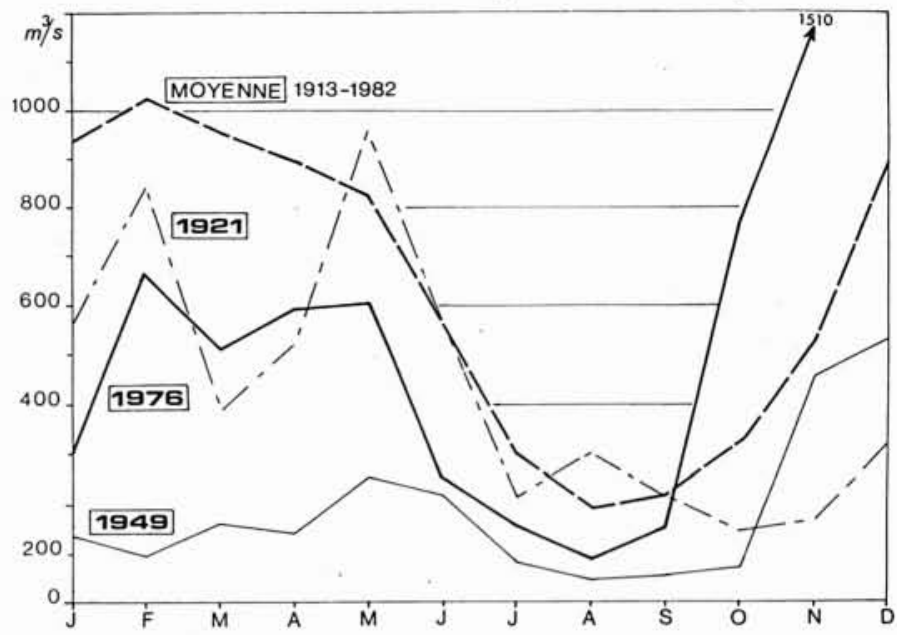

te bordegne à Eomme-sente

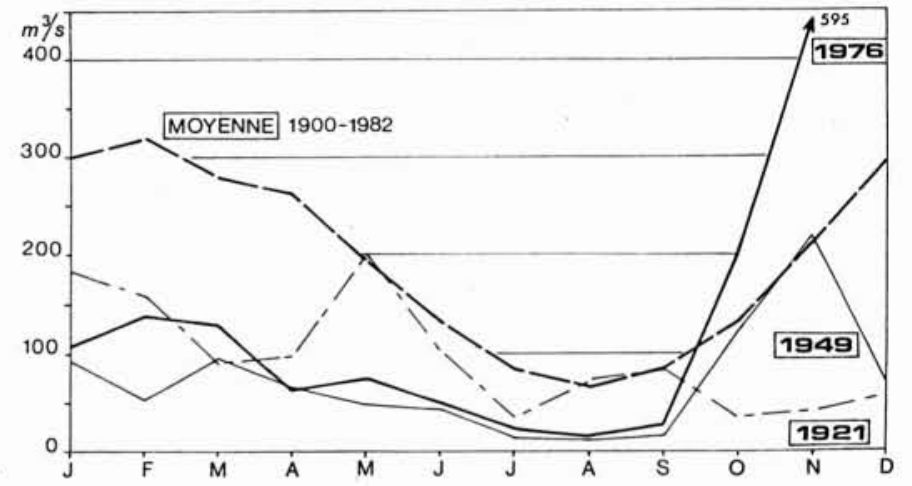

En France:

- la Durance observée depuis 1918 présente le plus faible écoulement annuel de la série d'observation avec un module de $90 \mathrm{~m}^{3} / \mathrm{s}$;

- la Dordogne à Domme-Cenac (cf. fig. 2) observée depuis 1900 connaît un module annuel de $94 \mathrm{~m}^{3} / \mathrm{s}(51 \%$ de déficit) qui figure au $2^{\mathrm{e}}$ rang de toute la série d'observation (seul celui de 1949 sera plus faible);

- la Garonne au Mas d'Agenais (station ouverte en 1913) connaît un déficit d'écoulement de $31 \%$ avec un débit annuel de $431 \mathrm{~m}^{3} / \mathrm{s}$ mais ce déficit n'a rien d'exceptionnel pour ce cours d'eau au régime hydrologique très irrégulier et qui a bénéficié en février et en mai (cf. fig. 2) de débits plus soutenus ;

- la Loire à Blois (observations continues depuis 1863) accuse un déficit de $57 \%$ qui le place au $2^{\mathrm{C}}$ rang de la série, mais la figure 3 montre bien par l'examen des débits annuels, que les étiages d'été n'ont rien d'exceptionnel alors que par contre la persistance de faibles débits jusqu'à l'hiver $21 / 22$ est tout à fait spectaculaire.

\subsubsection{La sécheresse de 1949}

On sait que la décennie 40 est très connue par ses sécheresses (hivers souvent froids et secs, suivis d'étés caniculaires) donnant une succession d'années largement déficitaires sur le plan de l'écoulement et des étiages d'été très faibles. Ainsi 43-44 est remarquablement sec en Angleterre, 45 en Italie, 47 en Allemagne (le Rhin à Lobith a les plus faibles débits sur 30 jours consécutifs avec $683 \mathrm{~m}^{3} / \mathrm{s}$ ).

En France, c'est l'année 1949 qui peut être considérée comme la plus sévère de cette décennie du point de vue hydrologique. Cette situation est provoquée par un hiver très sec suivi d'une période chaude et ensoleillée de mai à septembre.

Les figures 2 et 3 donnent l'exemple de 4 grands cours d'eau :

- l'hydrogramme annuel de la Loire à Blois est très aplati, les débits de juillet à octobre sont les plus faibles de ce siècle, ainsi que le module annuel qui connaît un déficit de $66 \%$ (le module interannuel est de $359 \mathrm{~m}^{3} / \mathrm{s}$ ); 


\section{le Lefre à Blote}

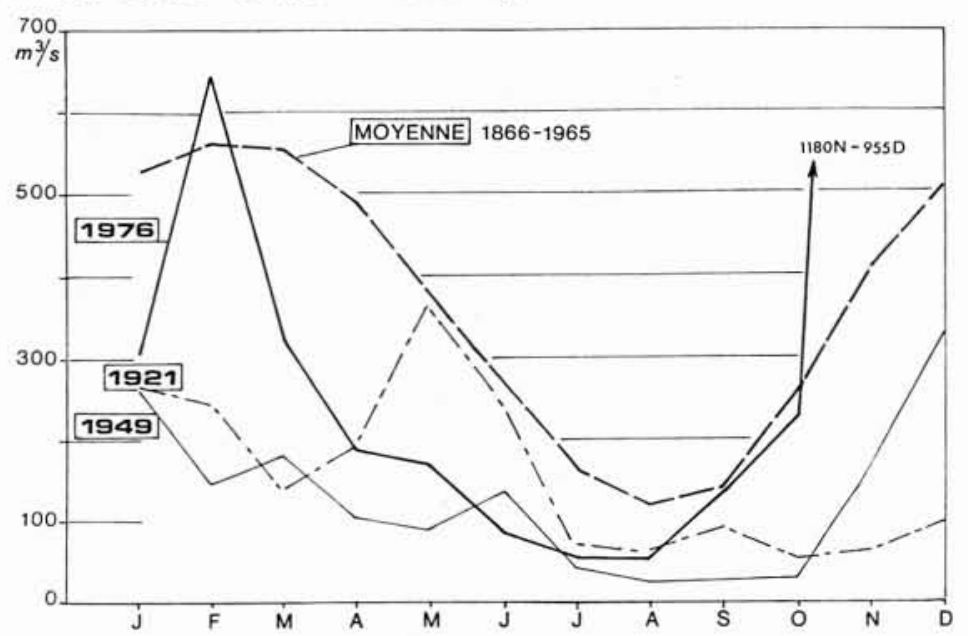

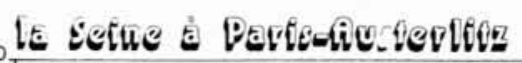

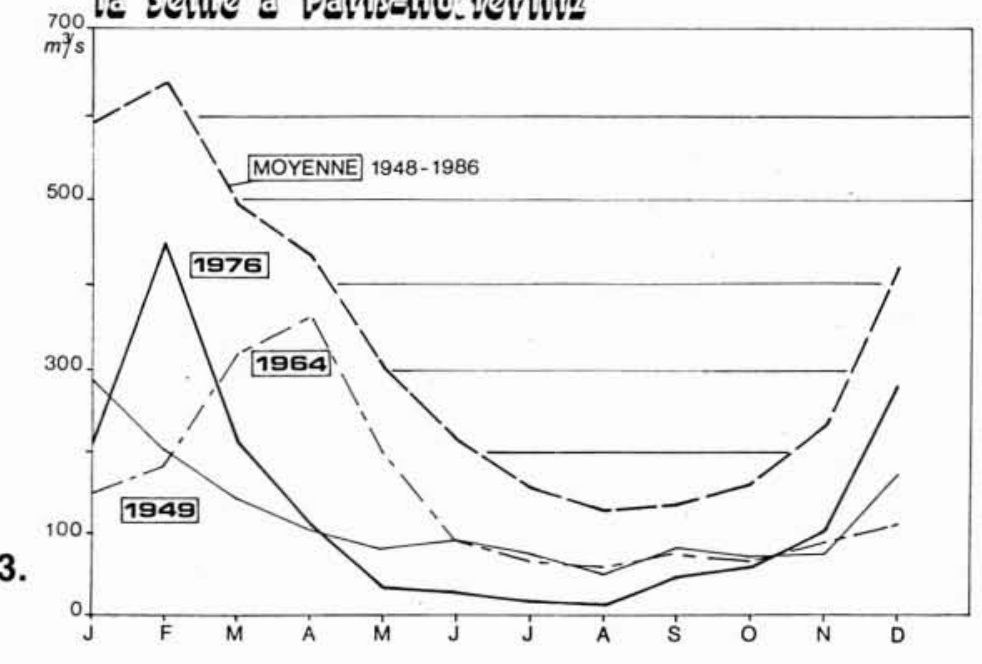

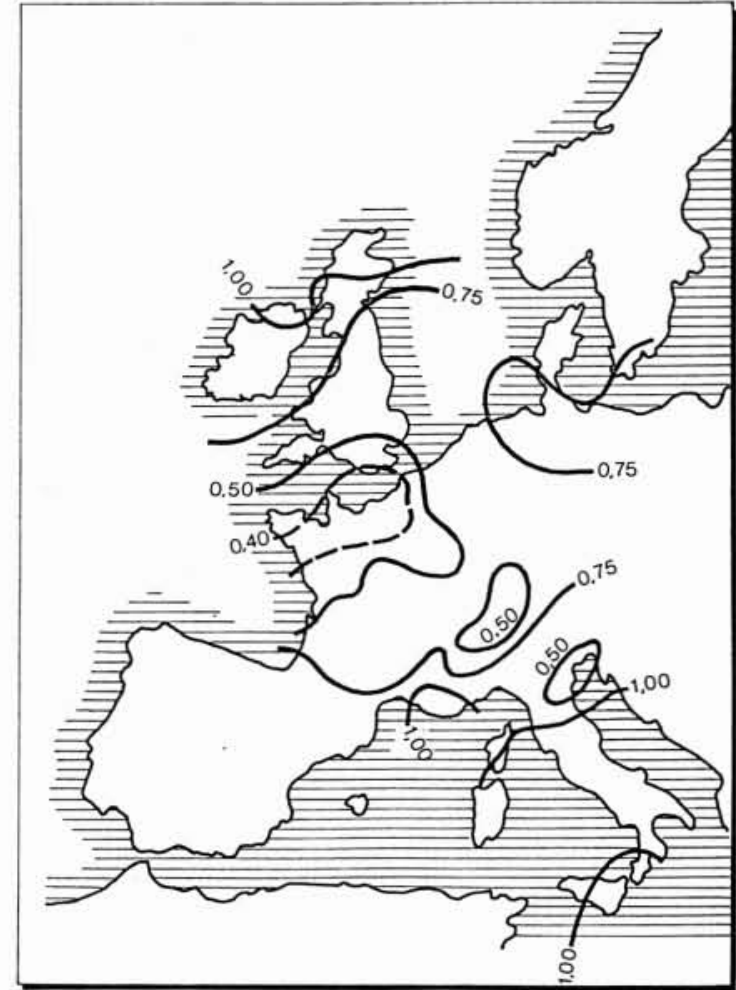

Rapport des pluies cumulées (1-12-1975 au 31-7-1976) Référence : normale 1931-1960 -D'après BROCHET-
- La Seine à Paris-Austerlitz (série en notre possession de 1948 à 1986) présente le plus faible module de cette série avec $102 \mathrm{~m}^{3} / \mathrm{s}$ mais les débits de mai à octobre seront très nettement plus faibles en 1976 ;

- la Dordogne à Domme-Cenac connaît : le plus faible module annuel de toute la série d'observations $69 \mathrm{~m}^{3} / \mathrm{s}$ (64\% de déficit), la plus faible série de débits moyens mensuels de février à septembre, avec un mois de février anormalement sec : $52 \mathrm{~m}^{3} / \mathrm{s}$, le plus faible débit minimal absolu avec $8,7 \mathrm{~m}^{3} / \mathrm{s}$ le 15 août ;

- la Garonne au Mas d'Agenais a un comportement analogue avec un déficit annuel record de $71 \%\left(187 \mathrm{~m}^{3} / \mathrm{s}\right.$ contre $635 \mathrm{~m}^{3} / \mathrm{s}$ ).

\subsubsection{La sécheresse de 1976}

Cet événement a laissé une empreinte qui reste présente dans les esprits. Il est comparable en sévérité à l'année 1921 mais a été moins prolongé puisque dès le mois de septembre le retour de pluies abondantes a pu gommer certains de ses effets. Il a montré que malgré le développement d'ouvrages régulateurs la ressource en eau de surface restait très vulnérable. Ce phénomène a bénéficié d'un suivi régulier du point de vue climatologique comme hydrologique et l'on dispose d'une littérature et de données chiffrées assez nombreuses.

\subsubsection{Aperçu pluviométrique}

La sécheresse commence début décembre et se fera sentir jusqu'à fin août avec des mois très déficitaires en décembrejanvier et mai-juin, le déficit hydrique étant aggravé par des journées très chaudes de la mi-juin à la mi-juillet.

Les figures 1 et 4 montrent que le maximum de déficit pluviométrique (50 à $60 \%$ ) se trouve sur l'ouest de la France et le sud de l'Angleterre (plus touchée qu'en 1921); l'Europe méditerranéenne est épargnée par ce phénomène (sauf le nord de l'Italie) alors que le nord de l'Europe est très atteint (minimum en 125 ans d'observations au PaysBas, minimum entre juin et septembre au Danemark depuis 1879 de même qu'au sud-est de la Norvège). En 


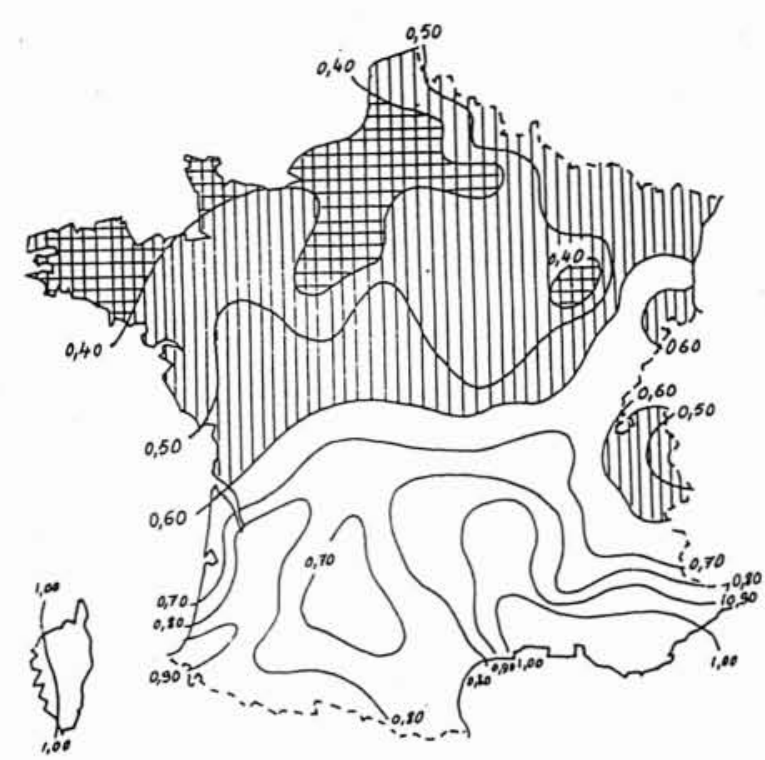

a. Précipitations cumulées du $I^{\text {er }}$ décembre 1975 au 31 août 1976. Rapports pluviométriques (Météorologie nationale).

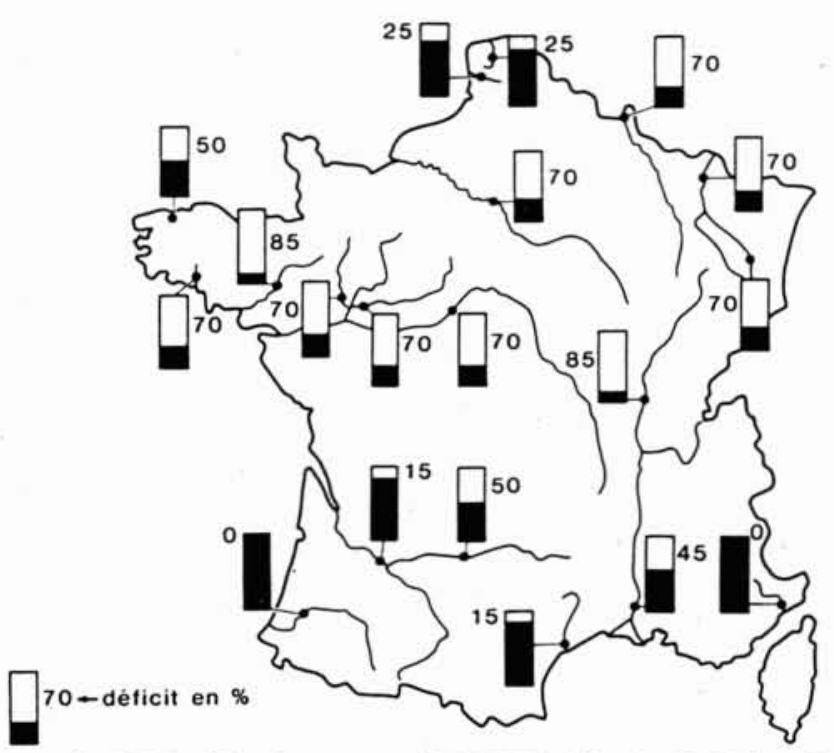

b. Déficit d'écoulement au 15-05-1976 (d'après G. Bédiot, La Houille Blanche, $n^{\circ}$ 6/7-1985).

5.

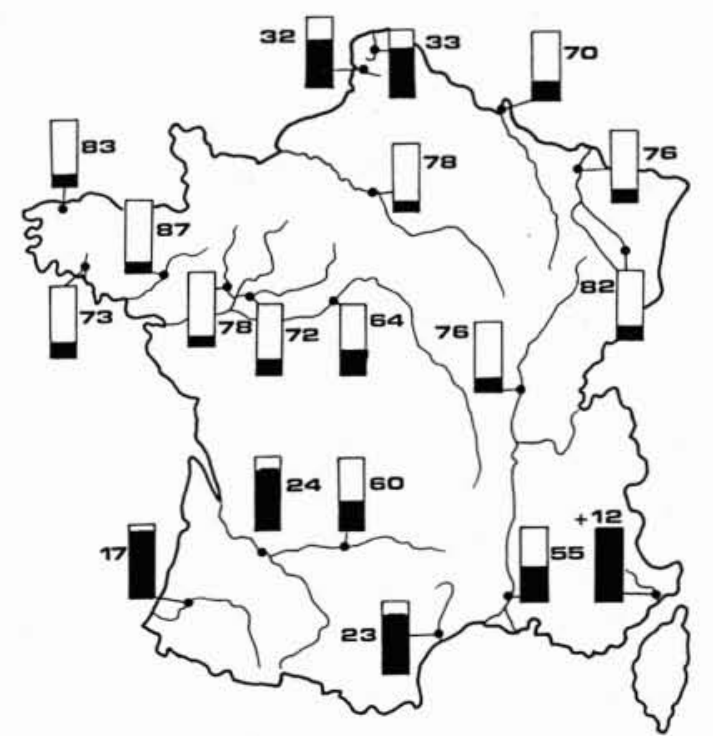

c. Déficit d'écoulement au 31-08-1976 en \% (cumul sur 5 mois).
RFA on assiste de même aux plus faibles précipitations aux mois de juin et d'août depuis le début du siècle.

La figure $5 a$ montre la situation en France: la moitié Nord est la plus affectée et seuls l'extrême sud-ouest et la Provence connaissent une situation pluviométrique conforme aux normales interannuelles.

\subsubsection{Aperçu hydrologique}

La figure $5 b$ donne les déficits d'écoulement pour une vingtaine de cours d'eau français à la mi-mai 1976. Le niveau des cours d'eau est déjà très bas et les déficits atteignent couramment $70 \%$ au nord de la ligne La Rochelle-Genève. Sur les bassins à dominance imperméable le déficit est exceptionnel (la Bretagne par exemple), mais sur les bassins soutenus à l'étiage par d'importantes réserves souterraines (l'Aa ou la Canche) le déficit reste modéré. Dans le sud du pays, l'Adour et le Var sont au contraire légèrement excédentaires.

La figure $5 c$ donne fin août la valeur des déficits d'écoulement (cumulés sur les mois d'avril à août) :

Les déficits sont en général encore aggravés; dans le nord de la France le soutien des fleuves côtiers par la nappe de la craie s'affaiblit. Au sud les déficits augmentent sensiblement, le bassin de l'Adour est modérément touché, sauf le Var qui présente un excédent atteignant $12 \%$.

Sur les petits cours d'eau les débits minimaux d'étiage sont parfois nuls (assèchement de la Vilaine à Cesson en juillet); le comportement des cours d'eau alpins est à cet égard intéressant : ceux à régime pluvio-nival ont une très faible hydraulicité alors que ceux à régime glaciaire voient leurs débits se maintenir grâce à une augmentation de la fonte des glaciers.

Sur les grands fleuves, les débits minimaux absolus ou à l'échelle du mois sont très faibles $\left(13,6 \mathrm{~m}^{3} / \mathrm{s}\right.$ pour la Seine à Suresnes en août) mais sont le plus souvent supérieurs * à ceux de 1949 .

- à l'échelle de 8 mois consécutifs (janvier à août inclus) c'est en général l'année 1949 qui est la plus sévère mais les valeurs 1976 en sont très proches, ainsi la Dordogne vient au $2^{\mathrm{c}}$ rang et la Garonne au $4^{\mathrm{C}}$ rang des valeurs classées; - à l'échelle du module annuel, la vigoureuse reprise de l'écoulement à partir d'octobre-novembre ôte beaucoup de signification aux chiffres obtenus.

Ainsi la Loire à Blois a une hydraulicité annuelle de 1,03 (module de $368 \mathrm{~m}^{3} / \mathrm{s}$ contre $359 \mathrm{~m}^{3} / \mathrm{s}$ en année moyenne). Le module annuel de la Garonne avec $563 \mathrm{~m}^{3} / \mathrm{s}$ (déficit de $11 \%$ ou hydraulicite de 0,89 ) ne vient qu'au $24^{\mathrm{e}}$ rang au non dépassement de toute la série d'observations et tous les modules annuels de la période 1942 à 1950 lui sont inférieurs.

A l'étranger, sur les grands cours d'eau déjà cités lors des sécheresses de 1921 ou 1949, on trouve également des valeurs très faibles, voire des records absolus à l'échelle d'un ou de 2 mois consécutifs mais à l'échelle de l'année les débits sont moins exceptionnels. Nous donnerons deux exemples :

- la Tamise à Teddington connaît sur 30 jours consécutifs 


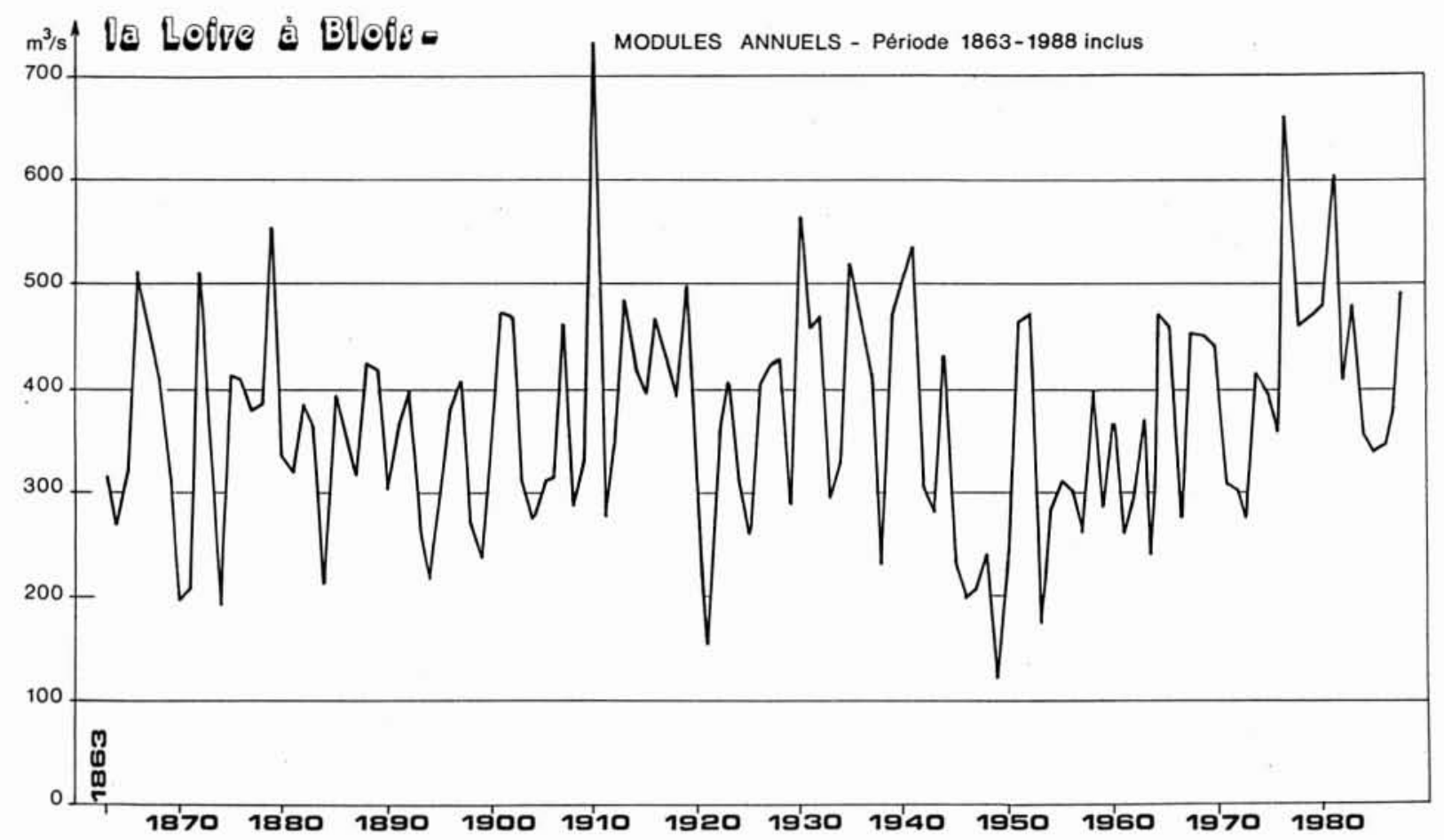

6.

le Danobe à Vieñe 1901-1980 - Modules annuels-

7.

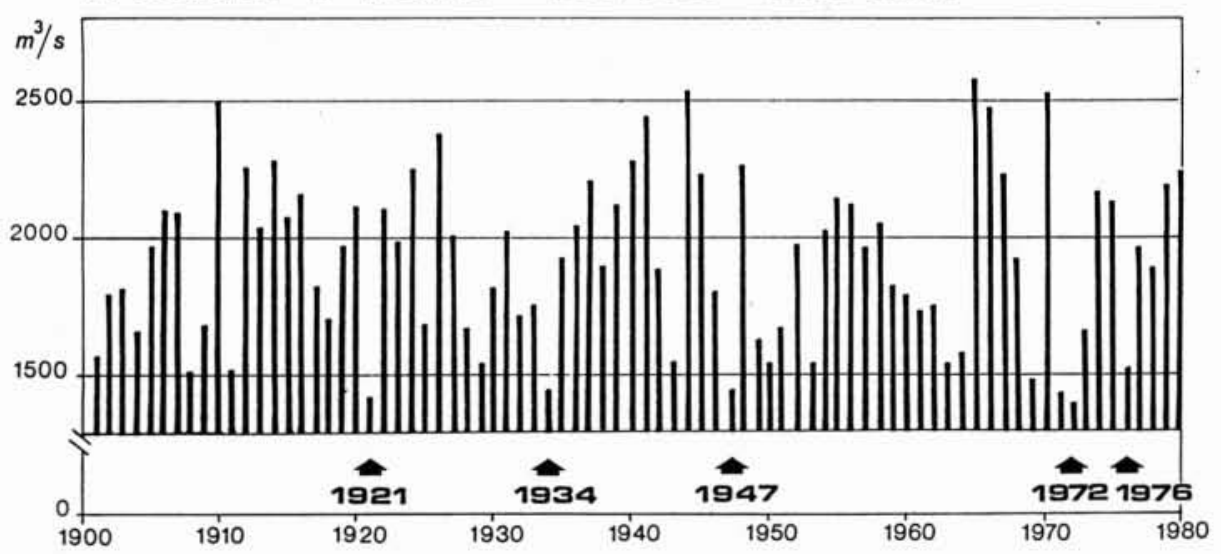

le plus faible débit sur presque un siècle d'observations. A l'échelle de 12 mois consécutifs (de septembre à août) le module vient au $3^{\mathrm{c}}$ rang de toute la série $\left(29,6 \mathrm{~m}^{3} / \mathrm{s}\right.$ contre $78 \mathrm{~m}^{3} / \mathrm{s}$ en moyenne) mais en année calendaire la période de retour serait à peine décennale;
- le Rhin à Lobith présente en juillet le plus faible débit mensuel observé avec $953 \mathrm{~m}^{3} / \mathrm{s}$ (contre $2180 \mathrm{~m}^{3} / \mathrm{s}$ en année moyenne, soit $56 \%$ de déficit) soit une période de retour proche du siècle. En année calendaire, le module 1976 ne vient qu'au $5^{\mathrm{c}}$ rang $\left(1333 \mathrm{~m}^{3} / \mathrm{s}, 40 \%\right.$ de déficit). 


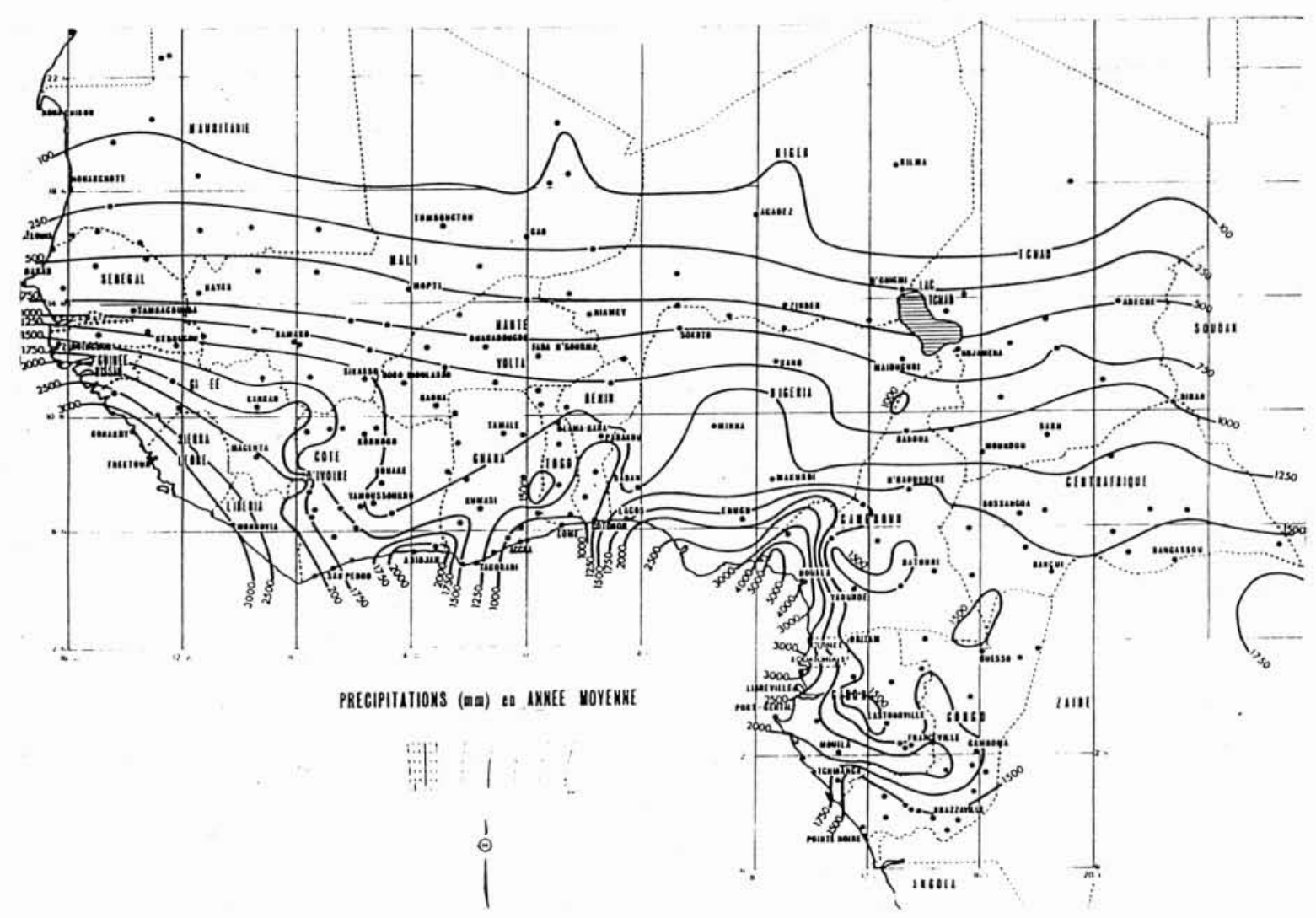

8.

\section{La sécheresse en zone sahélienne}

Dans cette courte présentation sur les sécheresses hydrologiques on aurait pu se limiter au seul cadre européen. Il a paru néanmoins utile de faire quelques remarques sur la situation en zone sahélienne où la persistance d'une sécheresse accusée depuis 1968 a entraîné des modifications spectaculaires de l'état des ressources en eau d'une zone couvrant plusieurs millions de $\mathrm{km}^{2}$.

\subsection{Historique}

Il est clair que les pays directement au sud du Sahara ont connu il y a plusieurs milliers d'années (comme le Sahara lui-même) des conditions climatiques plus humides; il suffit d'évoquer les peintures rupestres ou d'observer la morphologie des réseaux hydrographiques fossiles pour s'en convaincre.

L'utilisation des documents historiques et des traditions orales ne permet pas de remonter très loin dans le passé en ce qui concerne l'appréciation objective de périodes de sécheresse. Toutefois les chroniques arabes (Tarikhs de Tombouctou notamment) relatent qu'au $17^{\mathrm{e}}$ et $18^{\mathrm{e}}$ siècle un certain nombre de calamités et de famines ont affecté le bassin du Niger, il en est ainsi en particulier de la période 1680-1692, de celle de 1730-1750 dans la région de Tombouctou où une partie de la population fut décimée, puis à nouveau de 1790 à 1810 .

- Au $19^{\mathrm{e}}$ siècle les traditions orales sont recoupées par les récits des voyageurs, explorateurs, militaires qui parcourent ces régions (période très sèche de 1820 à 1840). Comme ailleurs, de véritables données chiffrées ne sont disponibles dans le meilleur des cas que depuis environ un siècle.

- En pluviométrie, les réseaux de base des pays sahéliens ne sont effectifs que depuis 1921-1922 mais il existe quelques stations plus anciennes, la "doyenne" étant celle de Saint-Louis du Sénégal (mesures de la pluie commençant en 1851).

- En hydrométrie les réseaux sahéliens ne débutent qu'en 1955-1960 mais sur les grands cours d'eau tropicaux les essais de navigabilité sur les fleuves commencent vers 18501860 et l'on dispose de séries complètes de hauteurs d'eau sur le Sénégal à Bakel depuis 1901 et sur le Niger à Koulikoro depuis 1907. 

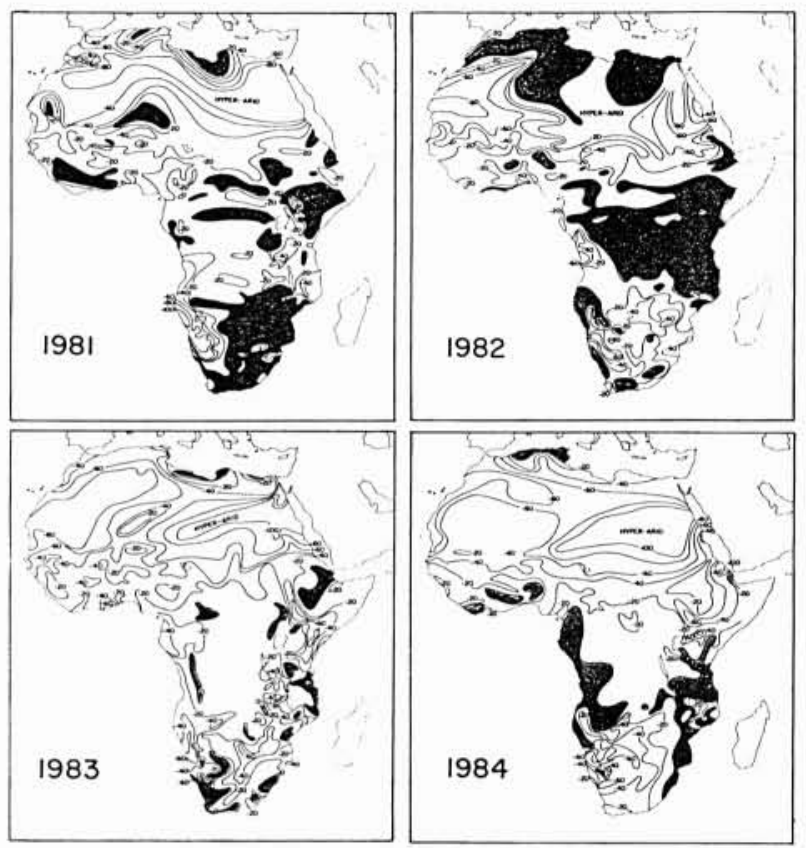
9. Precipitations de 1981 à 1984 - Ecarts à la normale (d'après
Nicholson)

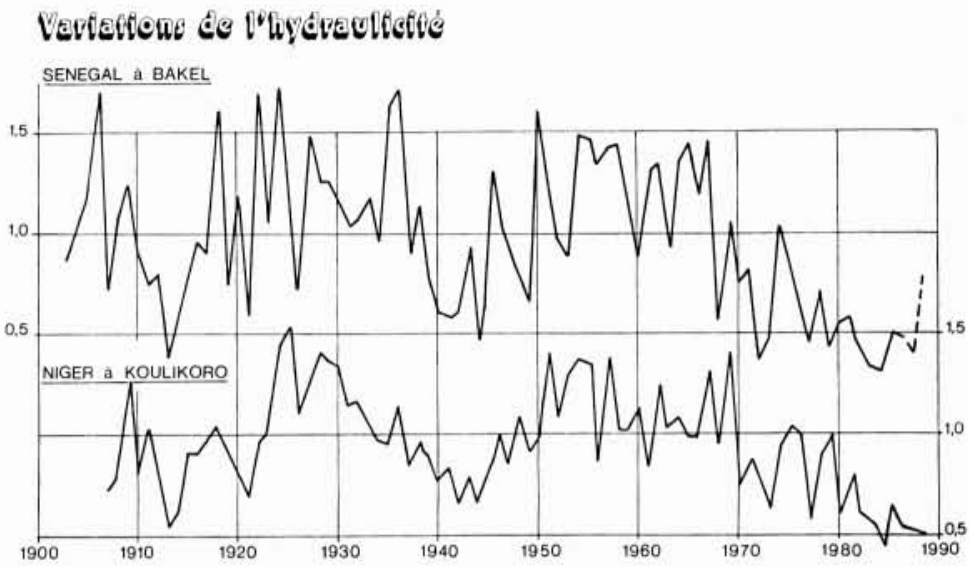

10.
- Au $20^{\circ}$ siècle, en dehors de la sécheresse actuelle, on note deux grandes vagues de sécheresse.

La première de 1910 à 1916 environ, culmine en 1913, elle présente la même extension que l'actuelle et a été considérée jusqu'en 1975-1976 comme la plus sévère de ce siècle.

La seconde, de 1940 à 1949, est caractérisée par une irrégularité spatio temporelle très forte, la diminution des ressources en eau se fait très nettement sentir mais les déficits sont globalement moins accentués que pour les deux autres sécheresses.

\subsection{La sécheresse 1968 à ce jour}

\subsubsection{Aperçu pluviométrique}

La sécheresse qui se maintient depuis 1968 en zone sahélienne avec des intensités variables mais toujours " déficitaires " jusqu’à présent, malgré l'amélioration de 1988, a été décrite par de nombreux auteurs. La figure 8 présente les isohyetes interannuelles (période 1920-1980 environ) sur toute l'Afrique de l'Ouest. Deux paroxysmes de sécheresse sont remarquablement sévères et étendus, celui de 1972 et 1973 au cours desquels de nombreux records absolus sont relevés avec des déficits atteignant entre les isohyetes 300 et $750 \mathrm{~mm}$ des valeurs de 30 à $60 \%$ et celui de 1982 à 1984 pendant lequel les minimas sont souvent à nouveau battus. La figure 9 (tirée de NiCHOLSON) montre les déficits pour ces années exceptionnelles.

\subsubsection{Quelques données hydrologiques}

La faible représentativité de l'information fournie par les cours d'eau sahéliens (hétérogénéité spatiale des pluies, variation des états de surface des sols) fait que la sécheresse hydrologique est beaucoup mieux appréciée par les grands fleuves tropicaux tels le Sénégal, le Niger ou le LogoneChari.

Modules annuels: La figure 10 montre les variations de l'hydraulicité des fleuves Sénégal et Niger. La longueur de leur période d'observation permettant d'apprécier l'importance des trois périodes de sécheresse survenues depuis le début du siècle. 


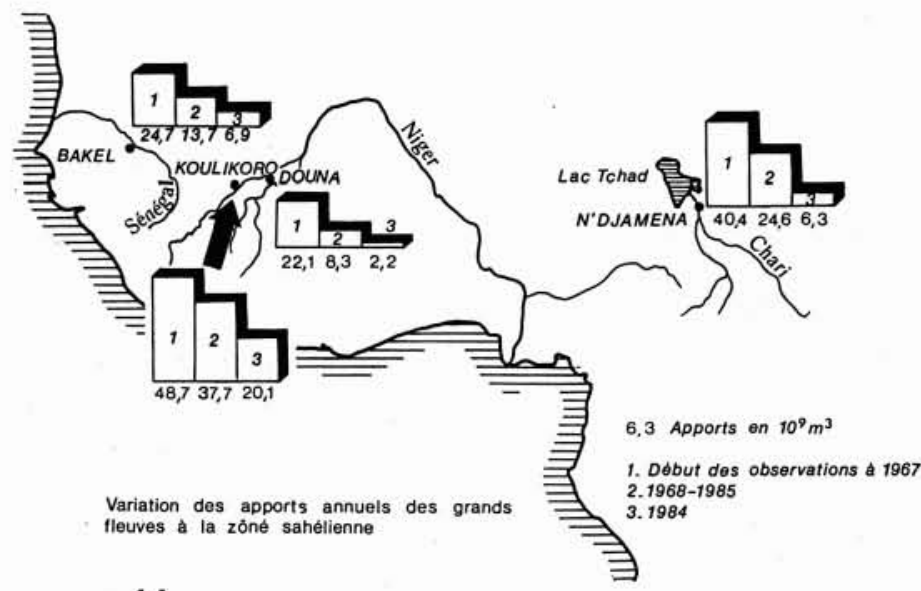

11.

Les tableaux ci-dessous récapitulent les valeurs annuelles les plus significatives.

\begin{tabular}{|c|c|c|c|}
\hline \multicolumn{4}{|c|}{$\begin{array}{l}\text { Sénégal à Bakel (1903-1988) } \\
\text { moyenne } 700 \mathrm{~m}^{3} / \mathrm{s}\end{array}$} \\
\hline Année & Module & Hydraulicité & Rang \\
\hline 1913 & 270 & 0,39 & 5 \\
\hline 1944 & 330 & 0,47 & 9 \\
\hline 1972 & 263 & 0,38 & 4 \\
\hline 1984 & 218 & 0,31 & 1 \\
\hline
\end{tabular}

\begin{tabular}{|c|c|c|c|}
\hline \multicolumn{4}{|c|}{$\begin{array}{l}\text { Niger à Koulikoro (1907-1988) } \\
\text { moyenne } 1430 \mathrm{~m}^{3} / \mathrm{s}\end{array}$} \\
\hline Année & Module & Hydraulicité & Rang \\
\hline 1913 & 810 & 0,57 & 5 \\
\hline 1944 & 990 & 0,69 & 13 \\
\hline 1973 & 930 & 0,65 & 12 \\
\hline 1984 & 636 & 0,44 & 1 \\
\hline
\end{tabular}

En terme d'apports annuels des fleuves tropicaux à la zone sahélienne, la figure 11 et le tableau ci-dessous montrent éloquemment l'ampleur du déficit qui règne depuis 1968 (valeurs en $10^{9} \mathrm{~m}^{3}$ ).

\begin{tabular}{|l|c|c|c|c|c|}
\hline Période & $\begin{array}{c}\text { Sénégal } \\
\text { à Bakel }\end{array}$ & $\begin{array}{c}\text { Niger à } \\
\text { Koulikoro }\end{array}$ & $\begin{array}{l}\text { Bani à } \\
\text { Douna }\end{array}$ & $\begin{array}{c}\text { Chari à } \\
\text { Ndjaména }\end{array}$ & Total \\
\hline $\begin{array}{l}\text { Début } \\
\text { des observ. }\end{array}$ & 22,3 & 46,2 & 17,3 & 35,1 & 120,9 \\
à 1985 \\
$\begin{array}{l}\text { Début } \\
\text { des observ. } \\
\text { à 1967 }\end{array}$ & 24,7 & 48,7 & 22,1 & 40,4 & 135,9 \\
$\begin{array}{l}1968-1985 \\
\text { Année 1984 }\end{array}$ & 13,7 & 37,7 & 8,3 & 24,6 & 84,3 \\
& 6,9 & 20,1 & 2,2 & 6,3 & 35,5 \\
\hline
\end{tabular}

Crues annuelles: En année normale les fleuves débordent largement de leur lit mineur et vont permettre, par irrigation ou submersion, le développement de vastes zones cultivées dans la basse vallée du Sénégal, le delta intérieur du Niger et les vastes plaines d'inondation du LogoneChari ; lors des périodes de sécheresse, la pointe annuelle de crue est souvent remplacée par plusieurs maximums peu marqués et de faible durée ayant un effet très médiocre sur les cultures. Depuis 1972 où les surfaces pouvant être irriguées s'étaient réduites de façon dramatique, la situation n'est jamais revenue à la normale et s'est à nouveau aggravée sensiblement depuis 1982.

\begin{tabular}{|c|c|c|}
\hline \multicolumn{2}{|c|}{ Sénégal à Bakel (1903-1984) } \\
\hline Année & $\begin{array}{c}\text { Crue maximale } \\
\text { moyenne } 4460 \mathrm{~m}^{3} / \mathrm{s}\end{array}$ & Rang \\
\hline 1913 & 1040 & 2 \\
1940 & 1740 & 5 \\
1972 & 1430 & 4 \\
1984 & 917 & 1 \\
\hline
\end{tabular}

\begin{tabular}{|c|c|c|}
\hline \multicolumn{2}{|c|}{ Niger à Koulikoro (1907-1984) } \\
\hline Année & $\begin{array}{c}\text { Crue maximale } \\
\text { moyenne } 5980 \mathrm{~m}^{3} / \mathrm{s}\end{array}$ & Rang \\
\hline 1913 & 3580 & 2 \\
1940 & 3940 & 6 \\
1972 & 3830 & 5 \\
\hline
\end{tabular}

Etiages absolus: La persistance de la sécheresse depuis 20 ans entraîne un appauvrissement catastrophique des nappes souterraines alimentant les cours d'eau en période de tarissement. La situation à la mi-1984 est analogue à celle observée à la mi-1974: le Sénégal à Bakel qui s'était totalement arrêté de couler en juin 1974 (événement inconnu jusqu'alors) s'est à nouveau tari fin mai 84 et fin mai 85. Depuis début 87 la mise en activité du barrage de Manantali a stoppé ce phénomène.

Sur le haut bassin du fleuve Niger la situation est également devenue très critique depuis 1968. Le fonctionnement du barrage de Selingue sur le Sankarani fait que les étiages à Koulikoro ne sont plus significatifs depuis 1981. Toutefois, malgré les lâchures de saison sèche, le Niger à Niamey s'est arrêté de couler en juin 1985 pendant quelques jours (phénomène jamais cité, même dans les Tarikhs).

\section{Conclusions}

Les sécheresses hydrologiques présentent, malgré leur altération progressive de la ressource en eau, des effets sérieux même en zone tempérée lorsque les besoins en eau ne sont plus temporairement satisfaits et peuvent perturber 
pendant plusieurs semaines ou plusieurs mois les activités économiques de pays entiers. En zone sahélienne la sévérité du phénomène et sa persistance entraînent des effets autrement plus dévastateurs que les inondations car affectant des surfaces énormes.

La maîtrise de l'eau dans ces régions sensibles comme la prévision du climat représentent des défis qui sont loin d'être résolus à l'aube du $21^{\mathrm{e}}$ siècle.

\section{Références bibliographiques}

BÉdiot G. (1985). - Faire face à la sécheresse. L'expérience française in La Houille Blanche numéro 6/7, 1985, p. 545557.

BÉRAN M.A., RODIER J.A. (1985). - Hydrological aspects of drought. UNESCO WMO. Studies and Reports in Hydro$\log \mathrm{n}^{\circ} 39,149 \mathrm{p}$.

BRÉMOND R. (1977). - La sécheresse exceptionnelle de 1976. $57^{\circ}$ congrès Montreux-Lucerne (14/6/77). Association générale des hygiénistes et techniciens municipaux, $14 \mathrm{p}$., 5 cartes, annexes.

Brochet P. (1977). - Aspects climatologiques de la sécheresse de 1976. IX Assemblée Plénière. Conseil supérieur de la météorologie, Paris, 6 p., 9 fig.

Bulletin de situation hydrologique du 15 avril 1989. Secrétariat d'Etat à l'Environnement. Service de l'eau. Sous Direction de l'Aménagement des Eaux, Neuilly, 9 p. + figures.

Climate System Monitoring. Monthly Bulletin WCP, n 12, 1988 and $\mathrm{n}^{\circ} 1,1989$, WMO Genève.

Evaluation of Drought in Czechoslovakia. Contribution to IHP. UNESCO Brastilava 1983.

Gerhard H. et al. (1983). - La sécheresse et les basses eaux de 1976. Commission Internationale de l'Hydrologie du Bassin du Rhin. Rapport n ${ }^{\circ} \mathrm{I} .2$ de la CHR, 81 p.

Howard F.M. (1979). - Hydrologic and Human aspects of the 1976-77 Drought, in Geological Survey Professional Paper 1130, Washington.
KRISHNAN A. (1979). - Key paper on definition of droughts and factors relevant to specification of agricultural and hydrologic droughts in hydrological aspects of droughts, 37 dec. 79, New Delhi.

Le Roy Ladurie E. (1983). - Histoire du climat depuis l'an mil. Collection Champs, Flammarion.

Météorologie (la). Numéro spécial "précipitations et hydrologie " $\mathrm{VI}^{\mathrm{e}}$ série $\mathrm{n}^{\circ}$ 20-21 mars et juin 1980

Mission interministérielle de l'eau. La sécheresse en 1976, aspects climatologiques, conséquences, actions administratives, propositions, $40 \mathrm{p}$.

Mounier J., Martin S., Marchand J.P. (1980). - Variations pluviométriques durant la période séculaire sur la façade occidentale de l'Europe. CNRS. Equipe de Recherche $\mathrm{n}^{\circ} 30$, Grenoble.

Rapport sur les effets de la sécheresse de 1976 sur la pêche (en Suisse), in Office Federal de la Protection de l'Environnement et de l'Inspection Fédérale de la pêche $n^{\circ} 38$.

RODier J.A. (1981). - Conférence sur les données hydrologiques extrêmes. Sécheresse et crues exceptionnelles. UNESCO Paris.

SERra L. (1960). - Caractéristiques et causes météorologiques des sécheresses. Fréquences d'apparitions in Conf. of surface water. (IAHS, Publ. $n^{\circ} 51$ ), p. 48-59, Helsinki, 1960.

Serra L. (1963). - Fluctuations de l'hydraulicité à l'échelle continentale in Symp. Eaux de surface, AISH. publ. $n^{\circ} 63$, Berkeley, p. 269-280.

SiRCoulon J. (1976). — Les données hydropluviométriques de la sécheresse récente en Afrique intertropicale. Comparaison avec les sécheresses « 1913 et 1940 ». In Cah. ORSTOM. sér. hydrol. vol. XIII, n², 76 p. 75-174.

Sircoulon J. (1986). - La sécheresse en Afrique de l'Ouest. Comparaison des années 1982-84 avec les années 1972-73. In Cah. ORSTOM, sér. hydrol. vol. XXI, n $4,1984 / 85$, p. $75-86$.

SirCoulon J. (1987). - Variation des débits des cours d'eau et des niveaux des lacs en Afrique de l'Ouest depuis le début du $20^{\circ}$ siècle. In AISH publ. $n^{\circ} 168$, Vancouver, p. 13-25.

Vivian H. (1979). - Climate and hydrologic droughts in Northern Alps in Hydrological Aspects of droughts. International Symposium, 3-7 dec. 79, New Delhi. 


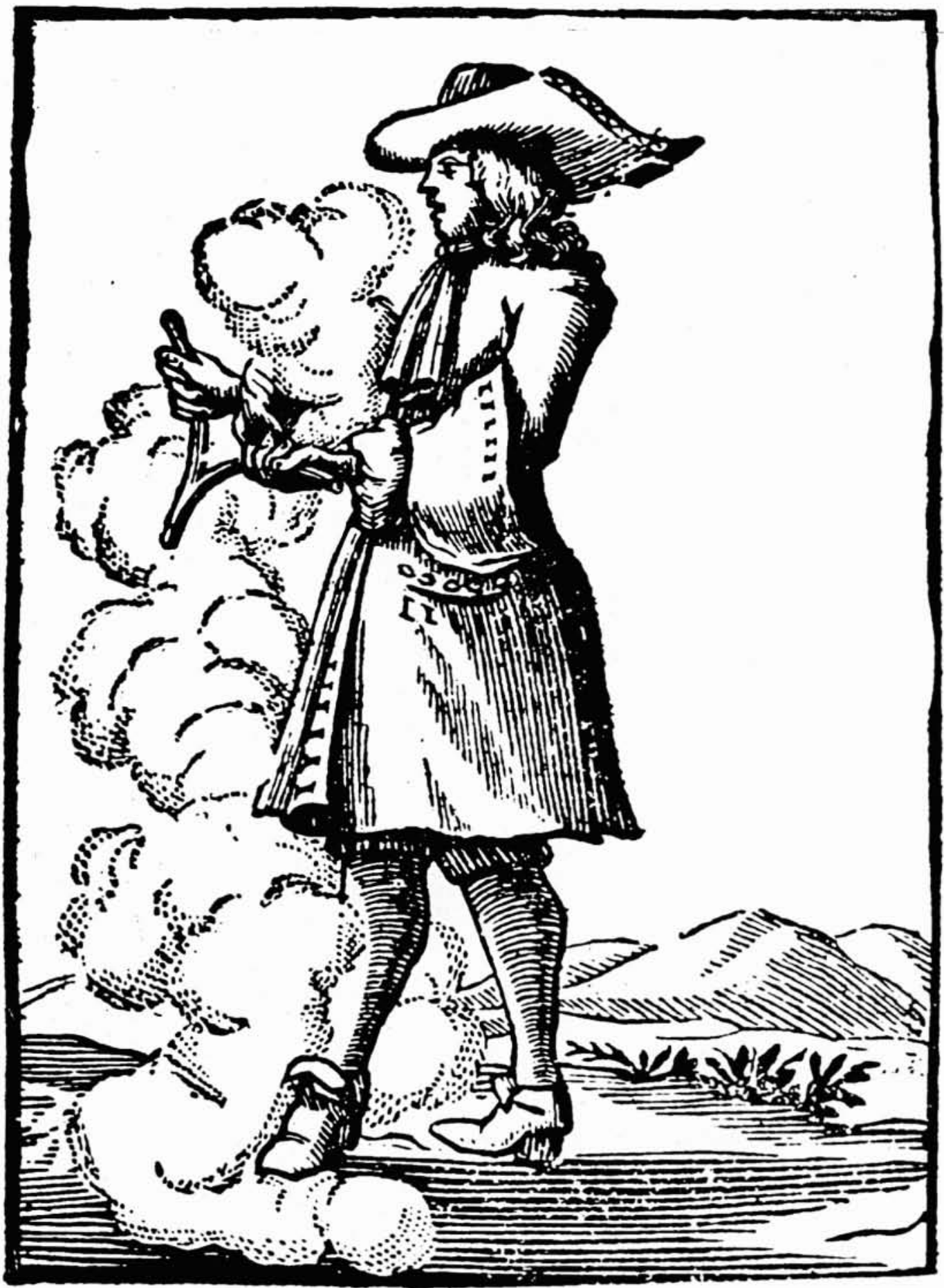

